\begin{tabular}{|c|c|}
\hline Title & $\begin{array}{l}\text { Ent r ai nment-enhanced Neural Osci Il at or for } \\
\text { I mit at i on Learni ng }\end{array}$ \\
\hline Author(s) & Yang, Wbosung; Chong, Nak Young; You, Bum Jae \\
\hline Citation & $\begin{array}{l}2006 \text { I EEE I nt er nat } \mathrm{i} \text { onal Conf er ence on I nf or mat i on } \\
\text { Acqui si t i on: } 218-223\end{array}$ \\
\hline Issue Date & 2006- 08 \\
\hline Type & Conf er ence Paper \\
\hline Text version & publ i sher \\
\hline URL & ht t p: //hdl . handl e. net /10119/9751 \\
\hline Rights & 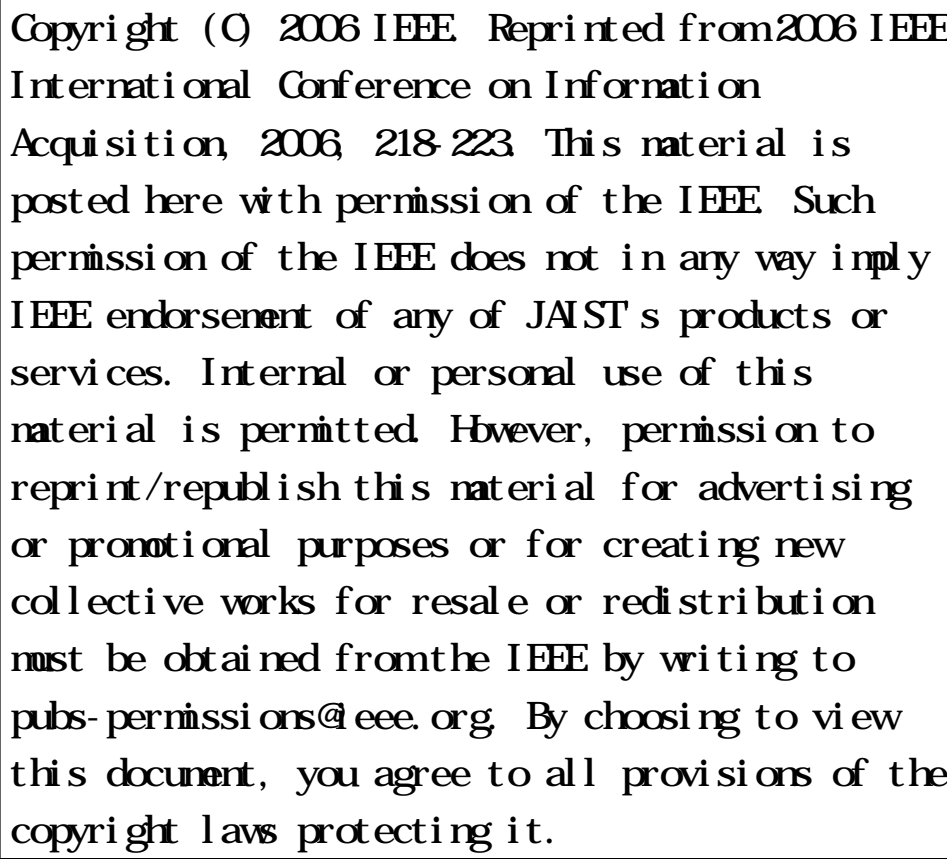 \\
\hline Des & \\
\hline
\end{tabular}

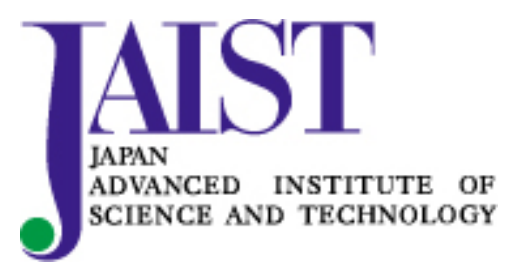




\section{Entrainment-enhanced Neural Oscillator for Imitation Learning}

\author{
Woosung Yang, Nak Young Chong \\ School of Information Science \\ Japan Advanced Institute of Science and Technology \\ 1-1 Asahidai, Nomi, Ishikawa 923-1292, Japan \\ \{woo-yang, nakyoung\}@jaist.ac.jp
}

\begin{abstract}
To achieve biologically inspired robot control architectures based on neural oscillator networks, goal-directed imitation is addressed with respect to the problem of motion generation. It would be desirable to easily acquire appropriate motion patterns for skill learning between dissimilar bodies to attain the goal of the demonstrated motion. This requires neural oscillator networks to adapt to the non-periodic nature of arbitrary input patterns exploiting their entrainment properties. However, even in the most widely-used Matsuoka oscillator, when an unknown quasi-periodic or non-periodic signal is applied, its output signal is not always closely entrained. Therefore, current neural oscillator models may not be applied to the proposed goal-directed imitation for skill learning. To solve this problem, a supplementary term is newly included in the equation of Matsuoka oscillator. We verify general properties of the proposed model of the neural oscillator and illustrate in particular its enhanced entrainment by numerical simulation. We also show the possibility of controlling dynamic responses of oscillator-coupled mechanical systems. Technical implications of the results are discussed.
\end{abstract}

Index Terms - Biologically inspired control, Neural oscillator, Entrainment, Imitation learning, Self-adjusting adaptor

\section{INTRODUCTION}

Most animals locomote perfectly well with inherent rhythmic movements in spite of differences in their sensors and actuators. It is known that these rhythmic movements are generated by central pattern generators (CPGs) in the spinal cord. Employing CPGs, that are neural networks producing rhythmic patterned outputs, we can realize the artificial nervous system for controlling locomotion in various types of legged robots. Across different groups of animals, entrainment plays a key role to adapt the nervous system to the natural frequency of the musculoskeletal system. This paper proposes a new model of artificial neural oscillator as a CPG featuring enhanced entrainment that facilitates the robot's skill learning by imitation. A neural oscillator coupled to the robot system incorporates sensory feedback of robot-environment interactions, allowing itself to deal with environmental perturbations. Therefore, the artificial neural oscillator gains increasing attention in the field of biologically inspired robotics that has proven to be deployable in uncertain realworld environments.

Matsuoka [1] proved that neurons generate the rhythmic patterned output and analyzed the steady state oscillation

\author{
Bum Jae You \\ Intelligent Robotics Research Center \\ Korea Institute of Science and Technology \\ 39-1 Hawolgok-dong, Seongbuk-gu, Seoul 136-791, Korea \\ ybj@kist.re.kr
}

conditions. He also investigated the mutual inhibition networks to control the frequency and pattern in the neural rhythm generator [2]. Taga [3] researched the entrainment properties between the rhythmic oscillation of the neural oscillator and the rhythmic locomotion of a musculo-skeletal system. Katayama et al. [4] addressed a nonlinear neural model with the harmonic balance method to design desired output patterns. Cao et al. [5] proposed the genetic algorithm based method to build up desired neural oscillator networks. Williamson [6] presented the neuro-mechanical system, where the artificial neural oscillator was coupled with the physical arm, and applied to robot arm control. Arsenio [7] suggested the multiple-input describing function technique to evaluate and design a nonlinear system based on the neural oscillator.

In this paper, the entrainment-enhanced neural oscillator (EENO) is proposed to improve existing neural oscillators. In order to make the EENO adapt to a quasi-periodic or nonperiodic input as closely as possible, a new term is added in the well-known mathematical model for the MNO. The EENO can be expected to be used for imitation learning between dissimilar robots that requires redesign of the perceived demonstrated behavior by the imitator robot. The modified behavior accommodates different kinematics and dynamics of the imitator and demonstrator robots. In the following section, we briefly describe the concept of goal-directed imitation. In Section III, we address the difference between the MNO and the proposed EENO. The detailed properties of both oscillator models are discussed in Section IV. Details of dynamic responses of both models coupled to a simple mechanical system are discussed in Section $\mathrm{V}$ and conclusions are drawn in Section V.

\section{GOAL-DIRECTED IMITATION FOR SKILL LEARNING}

The difficulties of learning by imitation lie in how to deal with the different kinematics and dynamics of imitator and demonstrator robots. The main idea of goal-directed imitation is to produce the same effect of the demonstrator robot's behavior by the imitator robot [8]. Note that the direction of the applying force should be coincident between two robots interacting with their environment. With this condition, the appropriate modified trajectory is acquired for the imitator. Then, comparing the perceived trajectory and the modified 
trajectory, the imitator can generate the compensated motion to achieve the intended goal of the demonstrated motion. Specifically, for biped locomotion, the direction of the reaction force at the point of foot contact should be coincident between two robots [9].

In our previous works [8-9], we have proposed the selfadjusting adaptor (SAA) through which the motion pattern acquired by the vision sensor was regenerated into an appropriate data from the point of view of kinematics adaptation. Different robots retained the same dynamic response by only adjusting their kinematic configuration, since all links were assumed to have a uniform mass distribution. For rigorous compensation of dynamic dissimilarities with non-uniform mass distribution between the imitator and demonstrator robots [10], the kinematically adjusted data enters the dynamics adaptor composed of a network of neural oscillators. The oscillator can be entrained with the input signal under a stable oscillation condition. That is to say, the SAA regenerates the perceived motion pattern into a new one adapted to the imitator's body. Through this sequence as is illustrated in Fig. 1, the regenerated motion data is mapped into the imitator's motion space. In practice, the dynamics adaptation process fundamentally relies on the entrainment capability of the neural oscillator. However, even in the most widely-used MNO, output signals are not always closely entrained with input signals if they exhibit non-periodicity. Thus, it is required to develop an enhanced artificial neural oscillator model that can entrain any non-periodic input signal.

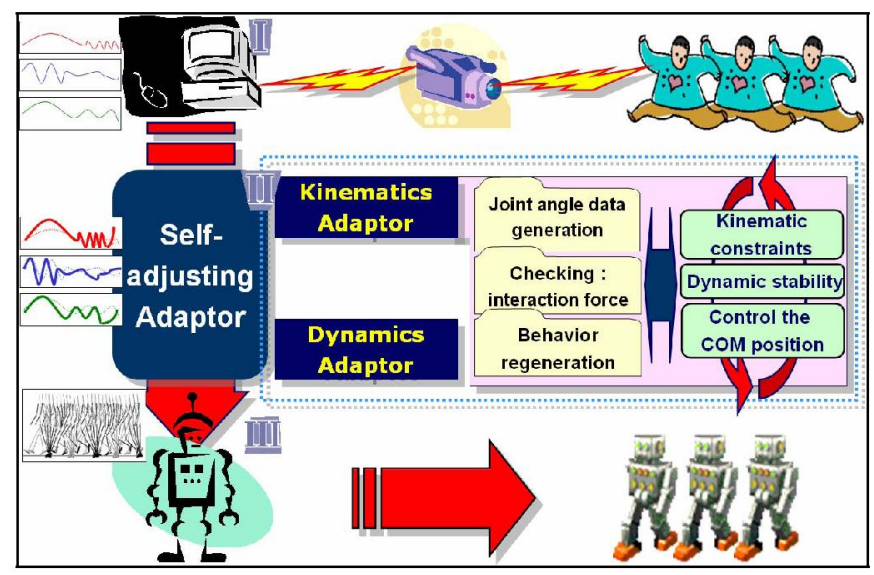

Fig. 1 Framework of motion imitation through the SAA

\section{Neural Oscillator Models}

The basic motor pattern generated by a CPG is usually modified by sensory signal from motor information to deal with environmental disturbances. Similarly, artificial neural oscillators are entrained with external stimuli at a sustained frequency. They show stability against perturbations through global entrainment between the neuro-musculo-skeletal systems and the environment [3]. Thus, neural oscillators have been applied to CPGs of humanoid robots with rhythmic motions [4], [11]. This section addresses the mathematical descriptions of the most widely-used MNO and the newly proposed EENO.

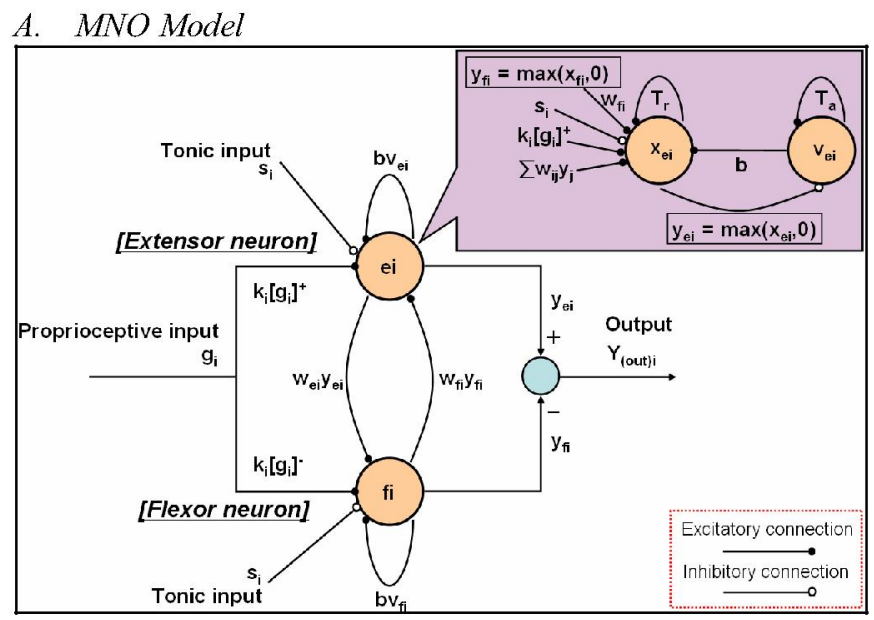

Fig. 2 Schematic diagram of the MNO

The MNO consists of two simulated neurons arranged in mutual inhibition as shown in Fig. 2 [1], [2]. If gains are properly tuned, the system exhibits limit cycle behavior. The trajectory of a stable limit cycle can be derived analytically and describes the firing rate of a neuron with self-inhibition. The MNO is represented by a set of nonlinear coupled differential equations given by

$$
\begin{aligned}
& T_{r} \dot{x}_{e i}+x_{e i}=-w_{f i} y_{f i}-\sum_{j=1}^{n} w_{i j} y_{j}-b v_{e i}-\sum k_{i}\left[g_{i}\right]^{+}+s_{i} \\
& T_{a} \dot{v}_{e i}+v_{e i}=y_{e i} \\
& y_{e i}=\left[x_{e i}\right]^{+}=\max \left(x_{e i}, 0\right) \\
& T_{r} \dot{x}_{f i}+x_{f i}=-w_{e i} y_{e i}-\sum_{j=1}^{n} w_{i j} y_{j}-b v_{f i}-\sum k_{i}\left[g_{i}\right]+s_{i} \\
& T_{a} \dot{v}_{f i}+v_{f i}=y_{f i} \\
& y_{f i}=\left[x_{f i}\right]^{+}=\max \left(x_{f i}, 0\right), \quad(i=1,2, \cdots, n)
\end{aligned}
$$

where $x_{e(f) i}$ is the inner state of the $i$-th neuron which represents the firing rate; $v_{e f(j) i}$ represents the degree of the adaptation, modulated by the adaptation constant $b$, or self-inhibition effect of the $i$-th neuron; the output of each neuron $y_{e(f ; i}$ is taken as the positive part of $x_{i}$, and the output of the whole oscillator is denoted as $Y_{\text {(oul) }} ; w_{i j} y_{i}$ represents the total input from the neurons inside a neural network; $w_{i j}(0$ for $i \neq j$ and 1 for $i=j)$ is a weight of inhibitory synaptic connection from the $j$-th neuron to the $i$-th, and $w_{e}$, $w_{f i}$ are also a weight from extensor neuron to flexor neuron, respectively; the input is arranged to excite one neuron and inhibit the other, by applying the positive part to one neuron and the negative part to the other; the inputs are scaled by the gains $k_{i} ; T_{r}$ and $T_{a}$ are time constants of the inner state and the adaptation effect of the $i$-th neuron respectively; $s_{i}$ is an external input with a constant rate.

\section{B. EENO Model}




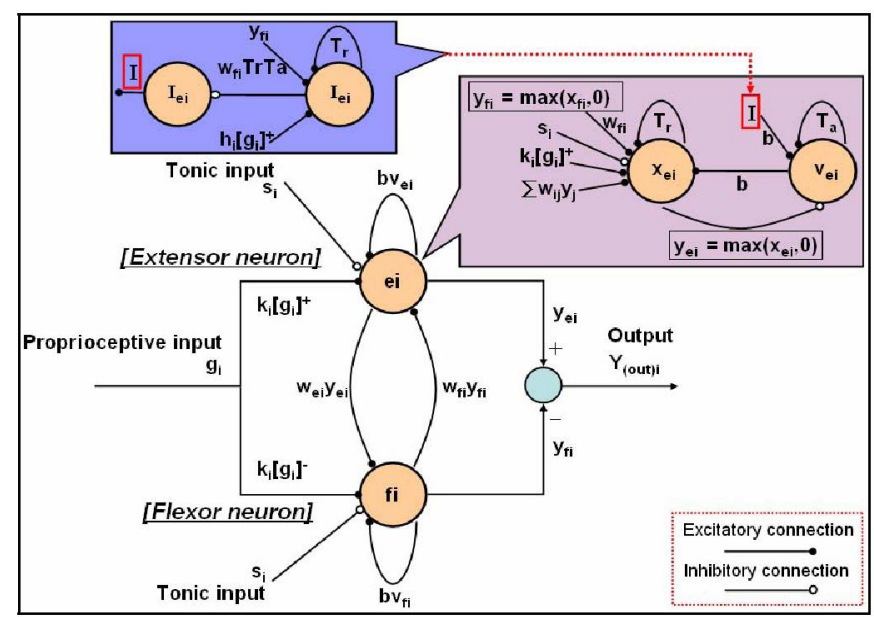

Fig. 3 Schematic diagram of the EENO

Technically, entrainment refers to tracking of sensory input signals, thus is very similar to the conventional feedback controller. Through mathematical manipulations of Eq. (1), we have found that the MNO functionally incorporated a proportional-derivative controller. It is straightforward to add an integral controller that eliminates the steady-state error in the MNO, which leads to the EENO given by

$$
\begin{aligned}
& T_{r} \dot{x}_{e i}+x_{e i}=-w_{f i} y_{f i}-\sum_{j=1}^{n} w_{i j} y_{j}-b v_{e i}-\sum k_{i}\left[g_{i}\right]^{+}+s_{i} \\
& T_{a} \dot{v}_{e i}+v_{e i}+b I_{e i}=y_{e i} \\
& T_{r} \dot{I}_{e i}=-T_{r} T_{a} w_{f i} y_{f i}-\sum h_{i}\left[g_{i}\right]^{+} \\
& y_{e i}=\left[x_{e i}\right]^{+}=\max \left(x_{e i}, 0\right) \\
& T_{r} \dot{x}_{f i}+x_{f i}=-w_{e i} y_{e i}-\sum_{j=1}^{n} w_{i j} y_{j}-b v_{f i}-\sum k_{i}\left[g_{i}\right]^{-}+s_{i} \\
& T_{a} \dot{v}_{f i}+v_{f i}+b I_{f i}=y_{f i} \\
& T_{r} I_{f i}=-T_{r} T_{a} w_{e i} y_{e i}-\sum h_{i}\left[g_{i}\right]
\end{aligned}
$$

\section{MODEL VERIFICATION AND VALIDATION}

This section investigates the properties of selfentrainment of the MNO and the EENO. The entrainment and input-output properties of the oscillators are used to perform a variety of tasks with the same architecture, without any modeling of system or its environment [7]. According to Matsuoka's work [1], [2], the entrainment can be realized under stable oscillation conditions. For stable oscillations, if tonic input exists, $T / T_{a}$ should be in the range of 0.1 to 0.5 , for which the natural frequency of the oscillator is proportional to $1 / T_{r}$. And increasing the input gain, $k_{i}$, causes the output of neural oscillator to be entrained with the amplitude and natural frequency of the input signal.

\section{A. Rhythmic Patterned Output Generation}

First, we begin with the rhythmic pattern generation of the MNO and the EENO under stable oscillation conditions. Let $Y_{i l}$ and $Y_{i z}$ denote the extensor and the flexor output of both oscillators with a designated initial condition, respectively. Also let $Y_{i}$ denote the total output of both oscillators. Three cases are investigated with different initial positions. The initial positions of $Y_{11}, Y_{21}$, and $Y_{31}$ are set to $0.8,0.2$ and -1.2, respectively. The upper graph of Figs. 4 and 5 show the position and the velocity of $Y_{11}-Y_{12}, Y_{21}-Y_{22}$, and $Y_{31}-Y_{32}$ according to the initial condition. The lower graph of Figs. 4 and 5 represents the plot of the total output. From these results, we can verify that the properties of rhythmic pattern generation with the EENO are coincident with those of the MNO under given stable oscillation conditions. Fig. 6 shows the FFT and phase plane plots of the MNO and EENO under the same condition.

\section{1) Rhythmic patterned outputs of the MNO}

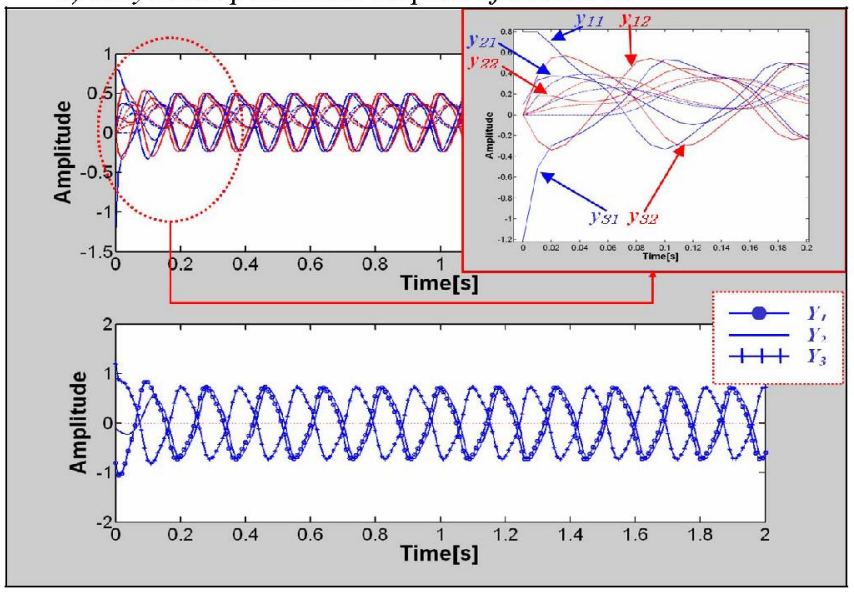

Fig. 4 The stable rhythmic patterned output of the MNO

\section{2) Rhythmic patterned outputs of the EENO}

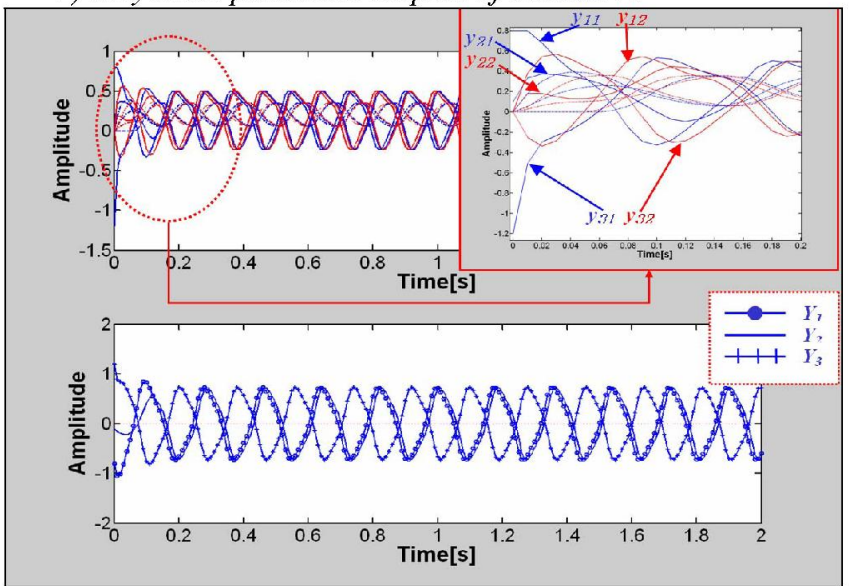

Fig. 5 The stable rhythmic patterned output of the EENO 

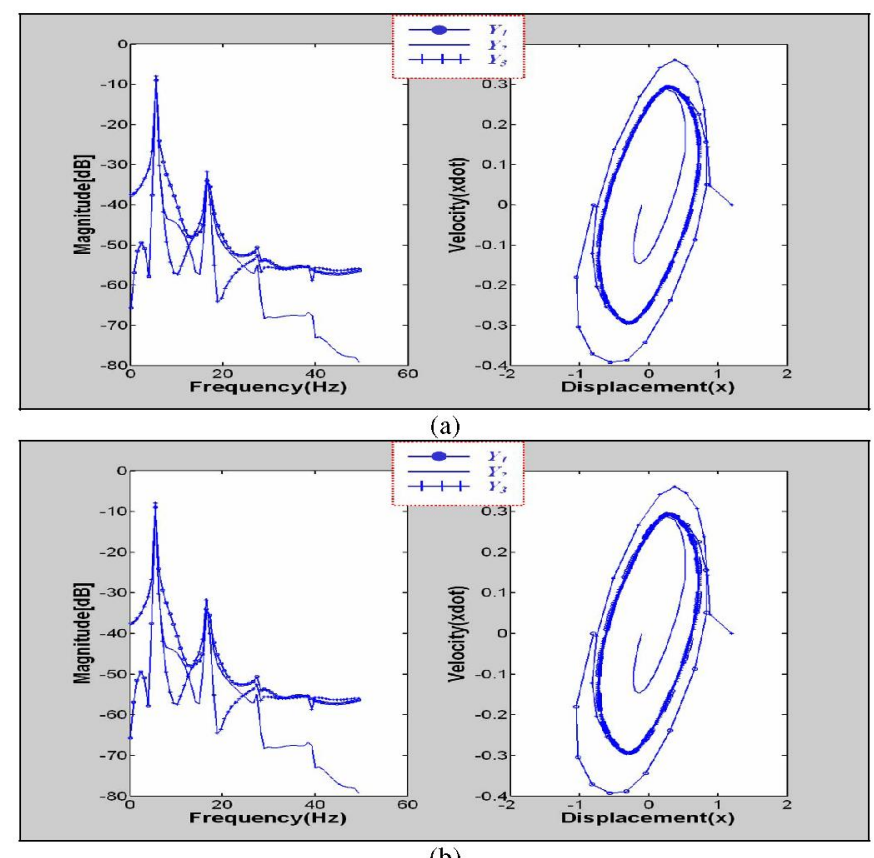

(b)

Fig. 6 (a) FFT (left) and phase plane (right) plots of the MNO (b) FFT (left) and phase plane (right) plots of the EENO

\section{B. Entrainment Properties}

This subsection addresses the entrainment properties of both oscillators. Note that we introduce the integral controller term which is expected to eliminate the entrainment error. Practically, the integrator term improves the degree of the adaptation or the self-inhibition effect. Tuning some other parameters of the equation as well, this entrained output signal can be controlled so that it follows the non-uniform multiphase input signal as closely as possible.

Generally, it has been known that the MNO exhibits the following properties: the natural frequency of the output signal increases in proportion to $1 / T_{r}$. The magnitude of the output signal also increases as the tonic input increases. $T_{r}$ and $T_{a}$ have an effect on the control of the delay time and the adaptation time of the entrained signal, respectively. Thus, as these parameters decrease, the input signal is well entrained. And the minimum gain $k_{i}$ of the input signal enlarges the entrainment capability, because the minimum input signal is needed to be entrained appropriately in the range of the natural frequency of an input signal. In this case, regardless of the generated natural frequency of the neural oscillator and the natural frequency of an input signal, the output signal of the neural oscillator locks onto an input signal well over a wide range of frequencies.

However, even though above-mentioned parameters are optimally tuned, the main limitation still exists in the MNO. If a quasi-periodic or a non-periodic input signal is entered into the oscillator, the MNO sometimes would not remain perfectly entrained as shown in Fig. 7. In this simulation, the values of $T_{r}, T_{a}$, and $k$ were set as $0.04,0.08$, and 0.6 , respectively. On the other hand, if we employ the EENO, the deviation from the input signal will disappear as shown in Fig. 8. Although the control parameter, $h$, of the integrator is not present here, it can be observed that the entrainment error decreases with time. With an optimally tuned parameter $h$, the entrainment is completely maintained, which is shown in Figs. 10. The result of Fig. 10 presents the comparison of an entrained signal with $h=0$ and $h=0.0011$ by the same input signal in the EENO. From the results so far, the EENO has proven to be robust with respect to the inputs of non-periodicity.

\section{1) Entrainment Results of the $M N O$}

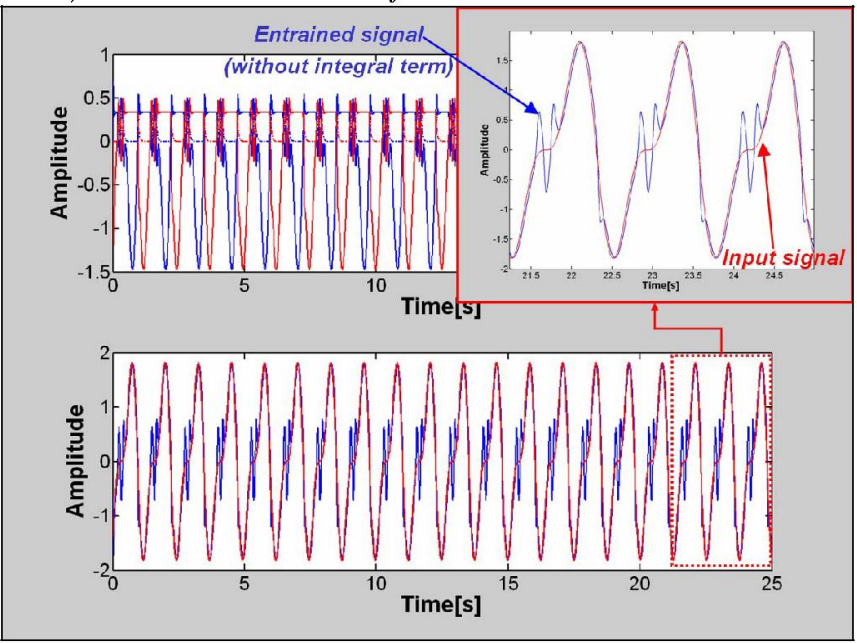

Fig. 7 The output signal of MNO entrained by the input signal

2) Entrainment Results of the Proposed Oscillator

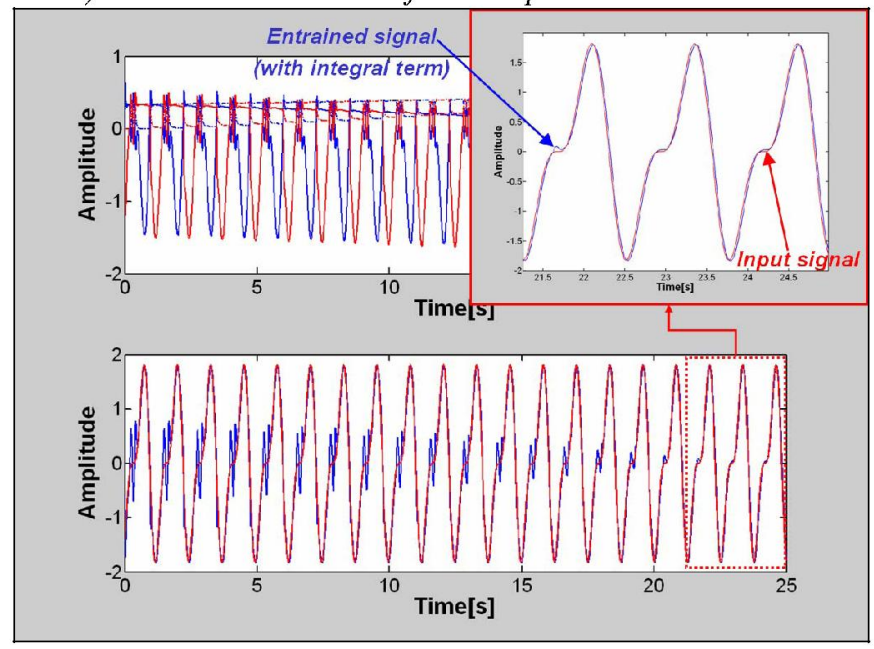

Fig. 8 The output signal of the EENO entrained by the input signal. 

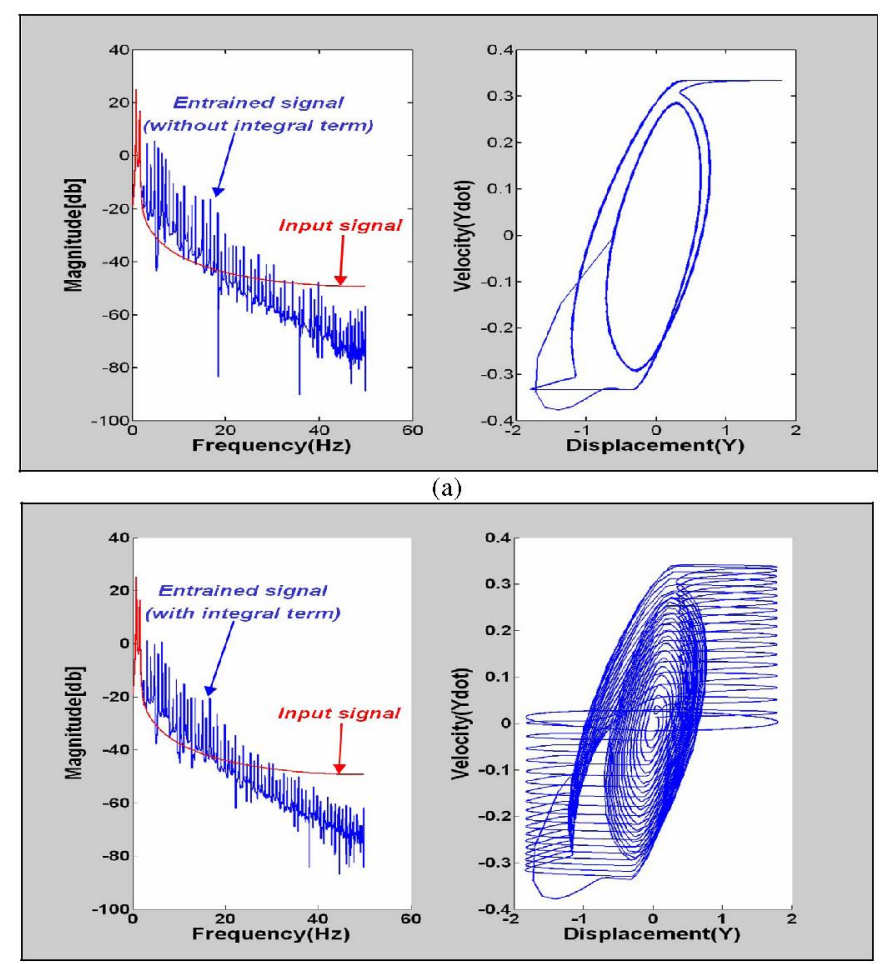

(b)

Fig. 9 (a) FFT (left) and phase plane (right) plots of the input and entrained signals of the MNO, (b) FFT (left) and phase plane (right) plots of the input and entrained signals of the EENO.

\section{3) Results of Integral Gain Change}

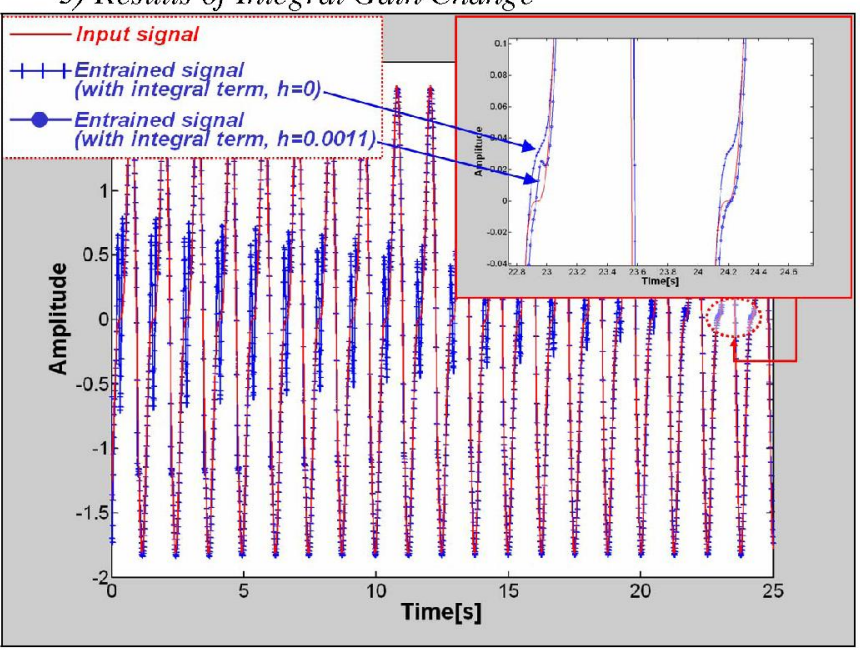

Fig. 10 The comparison of gained entrained signals of the EENO.

\section{DyNAMIC RESPONSE OF OSCILLATOR COUPLED MECHANICAL SYSTEM}

This section addresses a new control method exploiting the natural dynamics of the oscillator coupled to the dynamic system that closely interacts with the environment. This method enables the imitator robot to adapt to changing conditions. For simplicity, we employed a 2nd order mechanical system model connected to the neural oscillator as illustrated in Fig. 11. The desired torque input at the $i$ th joint can be given by [6]

$$
u_{d i}=k_{i}\left(\theta_{v i}-\theta_{i}\right)-b_{i} \dot{\theta}_{i},
$$

where $k_{i}$ is the stiffness of the joint, $b_{i}$ the damping coefficient, $\theta_{i}$ the joint angle, and $\theta_{v i}$ the equilibrium point which is the output of the neural oscillator. The output of the neural oscillator drives the mechanical system corresponding to the sensory signal input (feedback) from the actuator (displacement or torque). The oscillator entrains the input signal, so that the mechanical system can exhibit adaptive behavior even under the unknown environment condition. The key to implementing this method is how to incorporate the input signal's amplitude information as well as its phase information. Recall that the output of the MNO maintains the same phase as the input signal, but they may not be identical in amplitude.

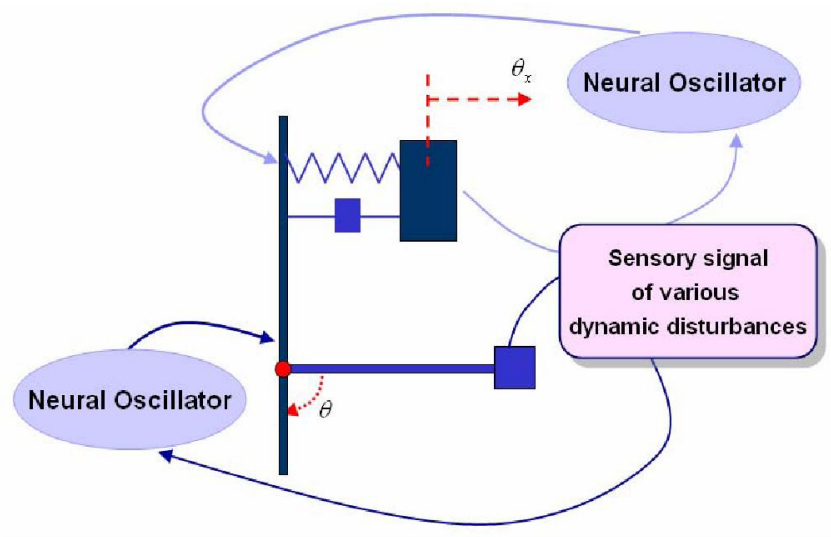

Fig. 11 Mechanical system model coupled to the neural oscillator.

We simulated the dynamic response to a sinusoidal input to compare the entrainment properties of the MNO and the EENO. In Fig. 12, the thick dashed lines are the outputs of the model that is not coupled to the oscillator, coupled to the MNO, and coupled to the EENO, respectively. Even though the frequency and phase are well entrained, the output amplitude is not identical in both the MNO and EENO models, if we compare them with the output of the uncoupled model. Theoretically, since the output is returned to the oscillator as the sensory signal input, the output should be adapted to the input, especially with the EENO coupled model. Note that Fig. 13 shows the good entrainment performance in the EENO coupled model, where the driving frequencies are $10 \mathrm{rad} / \mathrm{sec}$. We obtained the same results when the driving frequencies are 15 and $20 \mathrm{rad} / \mathrm{sec}$. Therefore, if the driving input frequency is far away from the natural frequency of the neural oscillator, it can not entrain the input signal with the same amplitude. We found that a proper range of frequencies exists to entrain the input signal completely even in the EENO coupled model. Therefore, one should carefully look into the range of possible frequencies to apply the EENO to the control of dynamic 
systems.

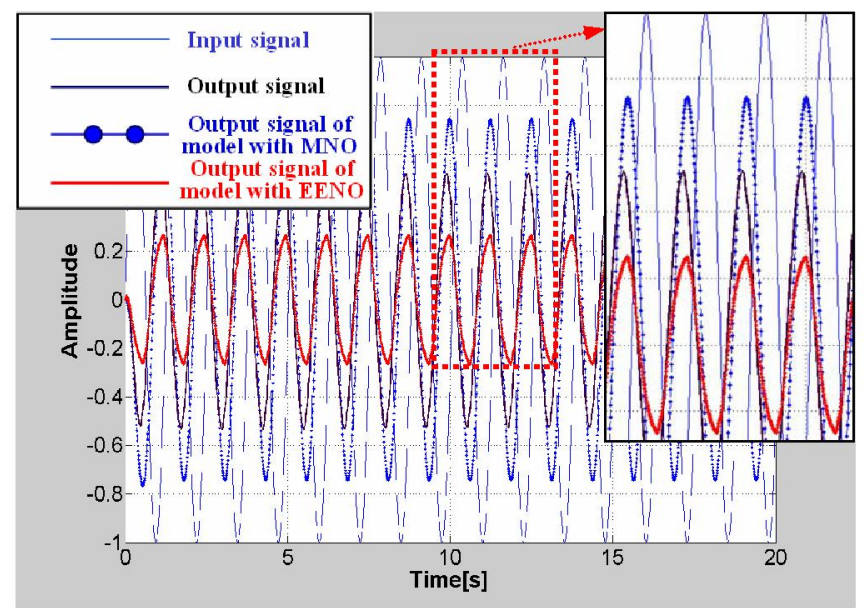

Fig. 12 Dynamic response of the MNO coupled and EENO coupled models with a $5 \mathrm{rad} / \mathrm{s}$ sinusoidal input

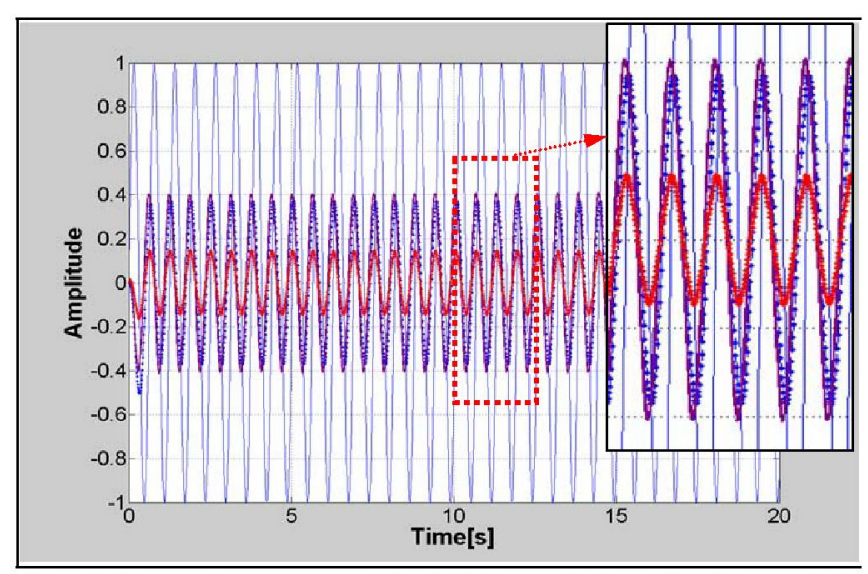

Fig. 13 Dynamic response of the MNO coupled and EENO coupled models with a $10 \mathrm{rad} / \mathrm{s}$ sinusoidal input

\section{CONCLUSIONS}

We have described a new model of the artificial neural oscillator with enhanced entrainment capability which can be applied to the goal-directed imitation for robot skill learning. Existing works on the neural oscillator did not address how to cope with the possible entrainment errors when the nonperiodicity of input signals was present. Our object was to develop a robust model of the neural oscillator with regard to uncertain inputs. For this, we newly introduced the integrator term in the mathematical model of the most widely-used Matsuoka neural oscillator.

The modified equation of the proposed neural oscillator was verified through extensive numerical simulations. Particularly, the entrainment properties of the proposed neural oscillator were investigated with different tuning parameters. Also, it was observed that the output signal of the proposed oscillator locked onto the unknown input signal with an optimally tuned parameter of the integrator. Specifically, the output signal of the proposed oscillator coupled mechanical system could be adapted to the frequency, phase, and amplitude of the sensory input signal over a reasonable frequency range. This approach is a new contribution toward realization of biologically inspired robot control architectures. A rigorous proof of the mathematical equation of the proposed oscillator is currently under way within the framework of control theory. Relating to future research, we will verify the practical validity of this approach through experiments with real robots.

\section{ACKNOWLEDGMENT}

This research is conducted as a program for the "Fostering Talent in Emergent Research Fields" in Special Coordination Funds for Promoting Science and Technology by Ministry of Education, Culture, Sports, Science and Technology of Japan.

\section{REFERENCES}

[1] K. Matsuoka, "Sustained Oscillations Generated by Mutually Inhibiting Neurons with Adaptation", Biological Cybernetics, Vol. 52, pp. 367-376 (1985).

[2] K. Matsuoka, "Mechanisms of Frequency and Pattern Control in the Neural Rhythm Generators", Biological Cybernetics, Vol. 56, pp. 345-353 (1987).

[3] G. Taga, "A model of the neuro-musculo-skeletal system for human locomotion", Biological Cybernetics, Vol. 73, pp. 97-111 (1995).

[4] O. Katayama, Y. Kurematsu and S. Kitamura, "Theoretical Studies on Neuro Oscillator for Application of Biped Locomotion", Proc. IEEE Int. Conf. on Robotics and Automation, Vol. 3, pp. 2871-2876 (1995).

[5] M. Cao and A. Kawamura, "A design method of neural oscillatory network for generation of humanoid biped walking patterns", Proc. IEEE Int. Conf. on Robotics and Automation, pp. 2357-2362 (1998).

[6] M. M. Williamson, "Rhythmic Robot Arm Control Using Oscillators", Proc. IEEE/RSJ Int. Conf. on Intelligent Robots and Systems, pp. 77-83 (1998).

[7] A. M. Arsenio, "Tuning of neural oscillators for the design of rhythmic motions", Proc. IEEE Int. Conf. on Robotics and Automation, pp. 1888$1893(2000)$.

[8] W. Yang and N. Y. Chong, "Goal-directed Imitation with Self-adjusting Adaptor Based on a Neural Oscillator Network," Proc. Int. Conf. on Advanced Robotics, pp. 404-410 (2005).

[9] W. Yang, N. Y. Chong, C. Kim, and B. J. You, "Locomotion Imitation of Humanoid Using Goal-directed Self-adjusting Adaptor", IEEE/RSJ Int. Conf. on Intelligent Robots and Systems, (2006), accepted for presentation.

[10]W. Yang and N. Y. Chong, "Dynamic Systems Control Using Entrainment-enhanced Neural Oscillator," Proc. Int. Conf. on Control, Automation, and Systems, pp. 1020-1024 (2005)

[11]M. Ogino, Y. Katoh, M. Aono, M. Asada and K. Hosoda, "Reinforcement learning of humanoid rhythmic walking parameters based on visual information", Advanced Robotics, Vol. 18, No. 7, pp. 677-697 (2004). 